the helicases have unwound the target, sequence-specific primers can bind to the single-stranded DNA and be extended, as in standard PCR.

Along with former colleagues, Kong has founded BioHelix Corporation in Beverly, with the aim of commercializing HDA. BioHelix's first commercial product is a teaching kit, marketed by the Carolina Biological Supply Company in Burlington, North Carolina, for students to carry out molecular diagnosis of sickle-cell anaemia in the classroom, without the need for an expensive thermocycler. The IsoAmp tHDA DNA amplification kit for research is due to launch this month.

\section{Primers and probes made easy}

For real-time PCR, the combinations of specific primers and the fluorescent oligonucleotide probes that detect template amplification are key. Researchers who hate designing probes and primers can now turn to online databases. PrimerBank, developed by Xiaowei Wang of the department of molecular biology at Harvard University and Massachusetts General Hospital, Boston, contains over 300,000 predicted primers for human and mouse genes generated computationally by a design algorithm. They have tested over 1,000 primer pairs and found a design success greater than $99 \%$ as defined by single PCR products and reasonable amplification efficiency, says Wang.

The Quantitative PCR Primer Database (QPPD), coordinated at the National Cancer Institute in Bethesda, Maryland, provides information about published primers and probes for quantitating human and mouse mRNA by RT-PCR.

Another source of published primerprobe sets for real-time PCR, with 3,376 entries so far, is RTprimerDB run by Jo Vandesompele and Filip Pattyn at the University of Ghent, Belgium, which can be searched by gene name, oligonucleotide sequence or NCBI's EntrezGene or SNP ID. "If people find the primer-probe pair they want they can click through to the PubMed identifier, and it also contains the details of the person who submitted the sequence," says Vandesompele. He hopes that coordinating the primer-probe sets that people use should increase standardization between labs, making it easier to compare results. Vandesompele also sees perils in the common use of a single housekeeper gene to normalize results in gene-expression studies, and his free geNorm applet for Excel will determine how many housekeeper genes you need for an accurate analysis. But he laments the fact that most analysis software only allows one housekeeper gene to be entered.

First described by Jesper Wengel in 1998, locked nucleic acids (LNA) are slowly making their way on to the PCR scene for use as probes. Their key advantage is their restricted conformational flexibility, which gives them great thermal stability and excellent mismatch discrimination when complexed with complementary DNA or RNA. At the Charité University Medical Centre in Berlin, Germany, Oliver Goldenberg and Lutz Hamann are using LNAs to quantify the speciesspecific $16 \mathrm{~S}$ rDNA from multiple bacteria in a single PCR reaction. Their interest is in the intestinal flora that keep us healthy and they want a fast method of detecting the bacteria present without having to cultivate them. With a standard reporter dye such as SYBR Green, which fluoresces when it binds doublestranded DNA, only one specific feature can be assayed per sample - either a total bacterial count or identification of a single species. The widely used fluorescent resonance emission transfer (FRET)-based probes, such as the TaqMan system from Applied Biosystems of Foster City, California, aren't suitable either, as they require a recognition sequence of some 40 bases and "you will hardly find such long conserved regions in 16S rDNA," says Goldenberg. "But the higher melting point of LNAs means that specificity can be achieved with shorter sequences."

Designing LNA probes can pose problems (see 'Simplifying the probe set'). "We have been testing LNA probes for SNP detection and have found the design parameters to be significantly different to standard TaqMan probes," says Helps. He thinks the problem lies in the probe-design software: the most commonly used design software was written for the established fluorigenic probes but doesn't work as well for LNAs.

PCR has its twentieth birthday this year and has stood the test of time. Like the DNA it analyses it is evolving, and the next 20 years should be equally exciting.

Pete Moore is a freelance writer based near Bristol, UK.

\title{
SIMPLIFYING THE PROBE SET
}

The conventional wisdom on probe-based real-time PCR assays says that if you have 10,000 genes to detect you will need 10,000 different probes to detect the PCR products. And to provide the required specificity, the probes need to be at least 18 nucleotides long. A team of researchers and bioinformaticians at Exiqon in Vedbaek, Denmark, is challenging this dogma. By scanning the genomes of key species they have come up with a set of 90 locked nucleic acid (LNA) 8- and 9-mer probes that they claim can be used to identify every gene on which you will ever work. Because of the greater

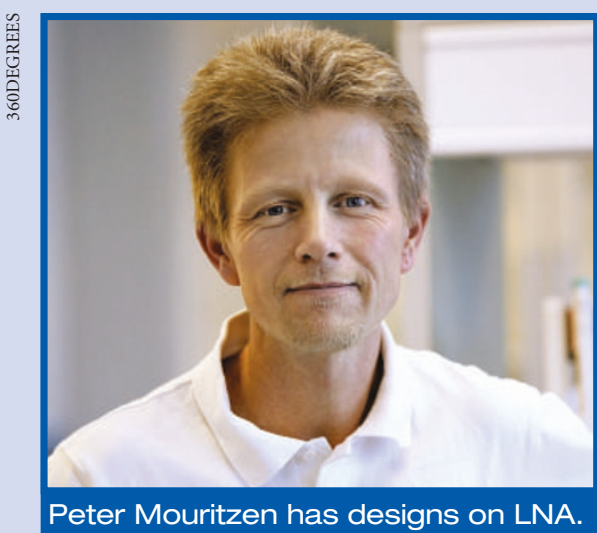

rigidity of LNAs, even a single mismatch with the potential target will seriously affect binding and prevent generation of signal, thus giving the same specificity as a longer regular oligonucleotide probe.

The probes come

to life when used with Exiqon's software, which for each target gene designs an optimum primer pair in combination with a specific probe. "The short LNA probes enable the technology, but the software is the key," says Exiqon's new technology-development manager, Peter Mouritzen. To identify the most specific combination, the software performs what Exiqon calls in silico PCR. This checks primer-probe predictions against the entire genome and transcriptome of the organism, minimizing the risk of possible mispriming which could produce a falsepositive signal from a real-time PCR assay.

For RT-PCR you want to look at the transcribed sequence and not the genomic DNA, which could contaminate the sample, so the software increases specificity by choosing a section where the primers span an exon-exon splice junction. While the primer could hit the genomic DNA, it will not generate a signal, because the intron is likely to make the product so long that it will not be amplified efficiently.

Users buy the LNA probes from Exiqon but can get their primers from any supplier. The assay design software is free online. "Normally people spend hours designing a PCR assay, and this thing does it in seconds," comments Mouritzen.

Roy Bicknell at the Weatherall Institute of Molecular Medicine at the University of Oxford, UK, agrees. "We look for new genes expressed on tumour vasculature as anticancer targets, so when we identify them by bioinformatics we have to do a lot of validation. Because we are looking at many genes we need to keep making new primers - and that is the beauty of the Exiqon system - it's for people who want to look at lots of different genes," he says. 